\title{
Thinking "Outside the Box": Unconstrained Creative Generation in Adults with Attention Deficit Hyperactivity Disorder
}

Creative generation is structured around existing knowledge and task examples, yet overreliance on specific examples may limit imagination (Ward, T. B., Finke, R. A., \& Smith, S. M., 1995). The constraining influence of knowledge during creative generation may be offset by conceptual expansion, a process of thinking outside traditional conceptual boundaries, which may be heightened by distractibility and mild executive dysfunction (Abraham, A., Windmann, S., Daum, I., \& Güntürkün, O., 2005). The present study explored the constraining effects of knowledge in adults with Attention Deficit Hyperactivity Disorder (ADHD), a disorder characterized by impaired attention and inhibitory control (Barkley, R. A., 1997). College students with and without ADHD were compared on two creative generation measures: an alien fruit invention task that required participants to imagine fruit that might exist on another planet (Ward, T.B., 1994), and a product label invention task wherein the goal was to invent product names without using elements of task examples (Kray, L.J., Galinski, A.D. \& Wong, E.M., 2006). ADHD participants created alien fruit that diverged more from Earth fruit and labels that conformed less to examples, relative to non-ADHD peers. Results suggest that adults with ADHD may be less constrained by knowledge during creative generation. Findings are discussed in terms of theoretical import and practical implications for individuals with ADHD.

Keywords: Attention Deficit Hyperactivity Disorder, conformity, creative generation, structured imagination, conceptual expansion, adults, chaotic cognition.

A motto among creative designers, "Never start at square one if you can start at square two," refers to the wisdom of using old models to inspire new creations (Goldschmidt, 2011). This philosophy may be applied to creative endeavors in general; whether elements of previous ideas or designs are deliberately reused or just serve as inspiration, no creation is entirely new (Ward, Finke \& Smith, 1995). Indeed, the application of old patterns to new designs, or the transfer of familiar structures to invention in unfamiliar domains, is illustrative of the role of analogical reasoning in creative endeavors (Casakin \& Goldschmidt, 2000; Ward, 2004). The guiding influence of knowledge on creative generation, or "structured imagination" (Finke, Ward \& Smith, 1992), is both efficient and practical. Yet, knowledge may also limit creativitythinking may get stuck or fixated on a particular idea (Finke et al., 1992). A real-world example is design fixation, wherein the creative process is stunted by interference from a specific example or perspective (Chrysikou \& Weisberg, 2005). In problem solving, prior exposure to old solutions may lead engineers to "fixate" on the previous solutions and reuse them in ways that are less effective (Jansson \& Smith, 1991). Experimental manipulations may reduce fixation and the constraining effects of knowledge during creative generation, however (Agogué et al., 2014; Chrysikou \& Weisberg, 2005; Marsh, Landau \& Hicks, 1996; Ward, Patterson \& Sifonis, 2004). Individuals also differ in the extent to which their imagination is structured (and thus constrained) by prior knowledge during creative generation (Abraham, Windmann, McKenna \& Güntürkün, 2007; Ward, Saunders \& Dodds, 1999). In particular, individuals with an overinclusive or chaotic cognitive style (Eysenck, 1993; Finke, 1996) may be less influenced by task examples or prior knowledge during creative generation (Abraham, Windmann, Daum \& Güntürkün, 2005; Abraham et al., 2007). The present study investigated structured imagination and creative generation in one such group of individuals: college students with Attention Deficit Hyperactivity Disorder (ADHD). The sections to follow provide a framework for this objective from a creative cognition perspective (Ward et al., 1995). 


\section{STRUCTURED IMAGINATION AND CONFORMITY IN CREATIVE GENERATION}

Creative generation is guided by, and structured around, knowledge (Finke et al., 1992). In the laboratory, structured imagination is observable using the alien invention task (Ward, 1994). The goal of this task is to create a variant of known category (e.g., animal or fruit), which might exist on another planet. Typically, participants first think of a known Earth-example of the category, which they then modify in various ways (Ward, 1994). Creative generation tends to start with the most accessible information (Finke et al., 1992). For instance, in the alien fruit task, participants are likely to draw inspiration from high frequency, typical of Earth fruits, such as apple or orange, which are easily retrieved from memory (Ward \& Wickes, 2009).Thus, creative generation follows a path of least resistance by default (Finke et al., 1992). The structuring influence of examples is often apparent in the resemblance between the creative product and its inspiration. Thus, alien fruit creations are likely to be juicy, sweet, and have seeds and a stem, because these features are typical of Earth fruit (Ward, Patterson, Sifonis, Dodds \& Saunders, 2002). Similarly, an alien creature inspired by an Earth animal is likely to show typical animal characteristics, such as bilateral symmetry (Ward, 1994).

In the absence of contextual information or task constraints, participants draw upon specific examples from memory during the alien creature invention task (Ward, 1994). In doing so, participants are likely to include more features typical of Earth animals. Yet, creations based on specific examples are less likely to include atypical features or attributes, and thus are lower in novelty (Kray, Galinsky \& Wong, 2006; Ward, 1994). Likewise, alien fruits that are inspired by specific Earth fruits are judged as less original (Ward et al., 2002). These studies demonstrate that when imagination is guided by knowledge, it may be constrained by that knowledge as well (Ward et al., 2002). Yet, experimental manipulations of structured imagination tasks may impact participants' reliance on specific examples during creative generation (Ward, 1994; Ward et al., 2004). By altering the constraints of the alien creature invention task, Ward (1994) directed participants toward either (a) retrieval of specific Earth animal exemplars, or (b) use of broader knowledge structures. Participants in the second condition were less likely to base creatures on specific Earth animals, and more likely to include novel (atypical) features in their invented creatures (Ward, 1994). Novelty is also higher when participants are instructed to use their wildest imaginations (Ward, 1994) or to consider factors that might impact survival on another planet (Ward et al., 2004). In the alien fruit invention task, reliance on specific Earth fruit exemplars is lower for participants instructed to not be bound by what fruit is like on Earth, compared to participants instructed to simply be creative (Ward et al., 2002). Thus, while creative generation tends to follows a path of least resistance by default, the creative approach is flexible and may change in response to task demands (Ward, 1994).

Creative generation is also influenced by recently activated knowledge in the form of task examples (Smith, Ward \& Schumacher, 1993). Smith et al. (1993) showed participants three example toys prior to a toy invention task. Each of these toys included three critical features: a ball, an electronic device, and a high level of physical activity. The effect of the examples was striking - despite explicit instructions to create toys that were very different from examples, the majority of participants included the shared attributes of the task examples in their toy designs (Smith et al., 1993). This tendency to retain features of examples in creative products, dubbed the "conformity effect" (Smith et al., 1993) is a robust phenomenon that has been observed in multiple contexts, using various experimental stimuli (Jansson \& Smith, 1991; Kray et al., 2006; Marsh, Ward \& Landau, 1999). Marsh et al. (1999) replicated the conformity effect using verbal materials, rather than visual. The product label invention task required participants to create labels for new products in each of three categories. In one condition, Marsh et al. (1999) provided task examples for each category which shared a common ending (e.g., pasta labels that ended in the letters "ni" — rotini, tortellini, rigatoni) and instructed participants not to copy the examples. Nonetheless, participants exposed to task examples were more likely to include example endings in the labels they created (Marsh et al., 1999).

Like schemas and self-generated examples, task examples may limit creative generation, as evidenced by lower novelty and originality of designs (Finke et al., 1992; Kray et al., 2006). Nonetheless, conformity per se does not necessarily imply that the creative process has been compromised or that the end product will be suboptimal (Marsh et al., 1996). In a study by Marsh et al. (1996), participants were exposed to various types of task examples prior to completing the alien creature invention task. As predicted by the conformity hypothesis, participants' designs included critical features of the examples, yet conformity had no effect on creative output, elaboration, or the inclusion of novel design features (Marsh et al., 1996). In fact, participants shown "artificial" examples (e.g., animals that possessed unusual feature pairings, such as claws and antennae) invented alien creatures that were rated as more creative, relative to inventions in the standard 
example and control conditions (Marsh et al., 1996). Notably, Marsh et al.'s (1996) artificial examples were highly original, which likely inspired relatively creative designs. Similar findings have emerged in problem solving studies; viewing unusual solutions prior a problem-solving task increases the originality of solutions (Agogué et al., 2014; Perttula \& Sipilä, 2007).

In an effort to explain the basis of the fixating versus inspiring ability of task examples, Agogué et al. (2014) crafted example solutions that exposed participants to either new or redundant information prior to problem solving. "Restrictive" examples were solutions expected to be spontaneously activated during problem solving, and therefore likely to reinforce the solver's conceptual problem space; "expansive" examples were solutions not expected to occur to the solver, and thus likely to widen the problem space. Agogué et al. (2014) observed that restrictive examples reduced the originality of participants' solutions, while expansive examples increased originality. On the basis of these findings, Agogué et al. (2014) concluded that the influence of a task example was determined by its effect on the solver's conceptual problem space; examples that widened the problem space increased originality, whereas examples that restricted or narrowed the problem space led to fixation (Agogué et al., 2014).

Studies that have varied parameters of creative generation and problem-solving tasks yield valuable information about conditions under which knowledge is more (or less) likely to constrain creativity. In the context of recently activated knowledge, unoriginal task examples are more likely to lead to fixation, and unusual task examples promote originality (Agogué et al., 2014; Marsh et al., 1996; Perttula \& Sipilä, 2007). Another way to reduce fixation is to interrupt the processes involved in structured imagination; this may be accomplished by adding an additional constraint, thereby blocking the path of least resistance (Landau \& Leynes, 2004). Youmans (2011) asked participants to complete a secondary task while viewing potentially fixating information; in this case, participants were too distracted to be influenced by the example in the first place. In a structured imagination task, such as the alien creature invention task, examples are retrieved from memory and creative generation is guided by participants' schemas (Ward, 1994). Reliance on self-generated examples can nonetheless be reduced, by encouraging an abstract approach (Ward, 1994) or varying instructions or task constraints in such a way that reliance on easily accessible or high frequency information is not a viable option (Ward, 1994; Ward et al., 2004). Collectively, these studies suggest that the constraining influence of knowledge during creative generation may be reduced by: (a) increasing conceptual expansion, and/or (b) disrupting the default path of least resistance or distracting from relevant information. As it happens, individual differences in cognitive processing may affect creative generation in much the same way (Finke, 1996).

\section{CHAOTIC, DIVERGENT PROCESSES IN CREATIVE GENERATION: THINKING OUTSIDE THE BOX}

The tendency to rely on knowledge during creative cognition may be universal, yet individuals differ in the degree to which they are constrained by knowledge during creative generation. Finke and Bettle (1996) describe two contrasting styles - ordered and chaotic - which are likely to impact structured imagination in opposite ways. Ordered cognition is linear, organized, and goal-directed (Finke \& Bettle, 1996). In the context of creative generation, ordered thinkers demonstrate a high degree of structure and connectedness to previous ideas and models (Finke, 1996). In contrast, the disorganized, spontaneous, and relatively unstructured style of chaotic cognition is likely to foster imaginative divergence, rather than structural connectedness (Finke, 1996; Finke \& Bettle, 1996). In many ways, chaotic cognition resembles the overinclusive processing style described by Eysenck (1993). According to Eysenck (1993), individuals differ in scope of perceived conceptual relevance, and people who define relevance more widely have an overinclusive processing style. In Eysenck's (1993) model, inclusiveness of processing is a key aspect of creative ideation. By virtue of broadly focused attention and conceptual activation, overinclusive thinkers are able to bring multiple ideas into close proximity, which facilitates original creative thinking (Eysenck, 1993). Eysenck (1993) predicted an overlap between the overinclusive processing style, trait psychoticism, and originality in divergent thinking, which was confirmed in later research (Acar \& Runco, 2012; Eysenck, 1994). In the context of creative generation, people with an overinclusive processing style may engage in relatively high conceptual expansion, a process whereby traditional conceptual boundaries are extended (Ward, 1994). Abraham et al. (2005) tested this hypothesis by comparing individuals with high trait psychoticism (high $-\mathrm{P}$ ) and individuals with low trait psychoticism (low- $\mathrm{P}$ ) on the alien creature invention task. As predicted, high-P individuals showed higher conceptual expansion on this task, as evidenced by the invention of alien creations that deviated more from Earth animals (e.g., lacked bilateral symmetry) compared to the creatures invented by low-P participants. Abraham et al. (2005) argued that the higher conceptual expansion in the high-P group 
was attributable to low latent inhibitory control and weaker top-down knowledge constraints. Abraham et al.'s (2005) findings suggest that the overinclusive, chaotic thinking associated with trait psychoticism may be beneficial for conceptual expansion during creative generation.

Chaotic cognition may likewise increase the ability to overcome the constraining effects of recently activated knowledge during creative generation (Abraham \& Windmann, 2008; Abraham et al., 2007; Eysenck, 1993; Finke \& Bettle, 1996). The information provided by task examples during creative generation is highly relevant and therefore not easily ignored (Abraham et al. (2007). Under these circumstances, attentional fluctuations and distractibility may give chaotic thinkers an edge (Eysenck, 1993; Finke \& Bettle, 1996). Studies of creative generation have found lower conformity to task examples among individuals with trait schizotypy (Abraham et al., 2007), patients with schizophrenia (Abraham \& Windmann, 2008), and children with ADHD (Abraham, Windmann, Siefen, Daum \& Güntürkün, 2006). Distractible individuals, such as children with ADHD, may be able to disregard salient knowledge by virtue of spontaneous activity in the default mode network (DMN), a group of structures deactivated during goal-directed activity (Beaty, Benedek, Silvia \& Schacter, 2016). Spontaneous activity in the DMN causes micro-lapses in attention which interfere with goal-directed activity (Sonuga-Barke \& Castellanos, 2007). In theory, these disruptions might also destabilize active knowledge patterns or distract from relevant examples during creative generation tasks, thereby reducing the constraining influence of knowledge on imagination.

\section{CREATIVE COGNITION IN ADHD}

ADHD is a neurological condition marked by distractibility, impulsivity, and hyperactivity that affects children and adults (American Psychiatric Association, 2013). ADHD is associated with deficits in attention, inhibitory control, and motivational regulation (Barkley, 1997; Castellanos \& Aoki, 2016; Nigg, 2001; Sonuga-Barke \& Castellanos, 2007; Sonuga-Barke, Sergeant, Nigg \& Willcutt, 2008). The cognitive profile of ADHD, while detrimental to activities which require organized, linear cognition, may nonetheless be beneficial for some aspects of creative cognition (Abraham et al., 2006; White \& Shah, 2006, 2011, 2016). In particular, adults with ADHD may be more original and innovative during divergent thinking (White \& Shah, 2006, 2016) and may show higher levels of creative achievement (White \& Shah, 2011). Creative cognition in ADHD may be partially attributable to deficits in executive inhibitory control (White \& Shah, 2006). This is consistent with the "less is more" role of prefrontal function in certain creative processes (ThompsonSchill, Ramscar \& Chrysikou, 2009). Neuroimaging studies have identified brain networks common to ADHD pathology (Castellanos \& Proal, 2012; Castellanos et al., 2008) and creative cognition (Beaty et al., 2016). Of particular relevance to creative generation is inefficient suppression of the default mode network, which has been linked to divergent creative thinking (Takeuchi et al., 2011) as well as ADHD (Fassbender et al., 2009; Sonuga-Barke \& Castellanos, 2007).

More broadly, creative differences related to ADHD may be framed in terms of chaotic cognition (Finke \& Bettle, 1996; White \& Shah, 2006). Like other chaotic thinkers, adults with ADHD are impulsive (Barkley, 1997), score high on divergent thinking measures (White \& Shah, 2006, 2011), prefer the idea generation phase of problem solving over clarification and development (White \& Shah, 2011), and find unusual uses for common, everyday objects (White \& Shah, 2006). In a recent study, adults with ADHD produced responses that were less semantically related to cues on a word association task, compared to non-ADHD peers (White \& Shah, 2016). Thus, adults with ADHD have a relatively wide scope of perceived relevance, as operationally defined by Eysenck (1993). On a cell phone feature invention task, White and Shah (2016) found that adults with ADHD invented features in more categories, compared to non-ADHD peers, and this difference was statistically mediated by semantic distance of responses on the word association test. White and Shah (2016) also found higher novelty for ADHD participants, compared to non-ADHD participants, as evidenced by invented features that diverged more from existing technology. These findings align with the predictions of Eysenck's (1993) model of creativity and overinclusive processing, and are consistent with greater imaginative divergence in adults with ADHD (White \& Shah, 2016).

By logical extension, ADHD might be expected to protect against the constraining effects of schema and self-generated examples during creative generation. Yet, Abraham et al. (2006) found no evidence of differences in conceptual expansion between adolescents with and without ADHD on the alien creature invention task. The null finding may have been due to a floor effect, however; as all participants in Abraham et al. (2006) scored low in conceptual expansion, relative to non-ADHD adults in another study that used the same task and scoring protocol (Abraham et al., 2005). The measurement technique may not have been ideal for assessing conceptual expansion; Abraham et al. (2006) used an aggregate measure that combined 
the presence of atypical features with a lack of typical features. However, studies have found ceiling effects for inclusion of typical features (e.g., Kray et al., 2006; Ward, 1994); in fact, even creatively gifted individuals are likely to include central attributes in their creations (Ward et al., 1999). Conceptual expansion may be more associated with the inclusion atypical features, rather than the exclusion of typical features.

Previous research has linked ADHD to divergent thinking (White \& Shah, 2006, 2011), which is a critical element of creative cognition (Ward et al., 1995). Yet, two aspects of creative cognition that have not been adequately assessed in ADHD are conceptual expansion and overcoming knowledge constraints (Abraham, 2014; Abraham \& Windmann, 2007). Neuroimaging research suggests that these components of creative cognition are dissociable from one another and from divergent thinking in terms of underlying pathways in the brain (Abraham, 2014). Overcoming knowledge constraints has been explored in the context of creative generation for children with ADHD, and in fact ADHD is associated with lower conformity to task examples (Abraham et al., 2006). Presumably, this advantage is related to distractibility, which may stem from DMN-interference (Abraham et al., 2006; Sonuga-Barke \& Castellanos, 2007). Yet, this phenomenon has not been examined in adults with ADHD, nor has the appropriateness of creations been evaluated in the context of a recently activated knowledge task for individuals with ADHD (in children or adults). Presumably by virtue of low latent inhibition and an overinclusive processing, individuals with high trait psychoticism show greater conceptual expansion during creative generation (Abraham et al., 2005). Adults with ADHD also have low latent inhibition (White, 2007) and an overinclusive processing style (White \& Shah, 2016), and should thus be less influenced by schemas and self-generated examples during creative generation. Abraham et al. (2006) did not find higher conceptual expansion among children with ADHD, perhaps due to floor effects and/or the young age of participants. Thus, the question of conceptual expansion in ADHD is yet unresolved.

\section{THE PRESENT STUDY}

The unique neurological profile associated with ADHD may protect against the constraining effects of knowledge during creative generation. Yet, with the exception of the study by Abraham et al. (2006), this hypothesis remains virtually untested. The present research addressed this gap by examining the well-established phenomena of structured imagination and conformity to task examples during creative generation in a sample of college students with and without ADHD. To this end, participants completed two creative generation tasks. The first was the alien fruit task, which required participants to draw and describe fruit that might exist on a planet very different from Earth (Ward et al., 1999, 2002). Participants were instructed to be as "creative and unusual as possible," which emphasized the creativity objective of the task (Ward et al., 2002). To create something unlike Earth fruit, participants would be compelled to think outside the traditional boundaries associated with the category "fruit." Evidence of this conceptual expansion is indicated by presence of atypical features (features that are not typical of Earth fruit) in alien fruit creations (Ward et al., 1999). The ADHD group was expected to show greater conceptual expansion, as indicated by the inclusion of more atypical features on the structured imagination task, relative to the non-ADHD group. The creations of the ADHD were also expected to be rated as more original, relative to the non-ADHD group, consistent with previous studies (White \& Shah, 2006, 2011).

The second creative generation task was the product label invention task (Kray et al., 2006; Marsh et al., 1999). This task required participants to invent labels for new products without using elements of the examples provided. On this task, overcoming knowledge constraints is indicated by lower conformity to task examples. The ADHD group in the present study was expected to include fewer elements of the task examples compared to the non-ADHD group; indicative of lower conformity during creative generation for individuals with ADHD. In addition, the present study measured the appropriateness of the product label inventions by rating the descriptiveness of invented labels. Past research suggests a trade-off between excluding example endings and descriptiveness ratings (Kray et al., 2006), yet White and Shah (2016) found that adults with and without ADHD generated equally appropriate cell phone features despite the higher novelty of features in the ADHD group. Thus, no predictions were made regarding possible group differences in label descriptiveness for the present study.

\section{METHOD \\ PARTICIPANTS}

Participants were undergraduate students at the University of Memphis $(N=52)$ enrolled in Introductory Psychology. The ADHD group $(n=26)$ included equal numbers of males and females, and half of the 
ADHD participants were taking stimulant medication at the time of the study. The non-ADHD group $(n=26)$ was also equal in gender composition. The ADHD and non-ADHD groups were similar in age $(M=20.4, S D=3.09 ; M=20.9, S D=3.65 ;$ ADHD and non-ADHD groups, respectively), as well as with respect to scores on the $\operatorname{ACT}^{1}(M=22.5, S D=3.49 ; M=21.8, S D=3.96$; ADHD and non-ADHD, respectively).

\section{PARTICIPANT RECRUITMENT PROCEDURE}

For participant recruitment purposes, a preliminary questionnaire was administered to approximately 800 students enrolled in Introductory Psychology at the University of Memphis as part of a large pre-screening session in which multiple researchers were recruiting participants for various studies. The questionnaire contained items from Barkley and Murphy's (1998) Current Symptoms Scale, as well as questions asking whether the individual had ever been clinically diagnosed with ADHD or any attention deficit disorder, and if so, whether the diagnosis had been confirmed by a clinician within the past 6 months. Of the pre-screening group, $48(\sim 6 \%)$ reported a diagnosis of ADHD and scored above the clinical threshold of the Current Symptoms Scale. Of the students who are qualified for the ADHD group on the basis of the initial screening, 28 agreed to participate in the study; of these potential participants, two students were excluded from the study due to comorbid psychiatric conditions (major depressive disorder, generalized anxiety disorder), which yielded a final sample of 26 participants for the ADHD group. ${ }^{2}$ An equal number of participants for the non-ADHD sample were drawn randomly from a large pool of candidates from the same subject pool, all of whom scored below the clinical threshold on the Current Symptoms Scale and reported no personal or family history of an attention deficit disorder, learning disability, or mood disorder. A second self-report measure, the Conners Adult ADHD Rating Scales, Screening Version (CAARS-S-SV; Conners, Erhardt \& Sparrow, 1999), was administered during the experimental session to confirm reliability of self-reported ADHD symptoms. Scores on the CAARS-S-SV were in the clinical range for the ADHD group, and in the nonclinical range for the non-ADHD group.

\section{MATERIALS}

Current and Childhood ADHD Symptoms Rating Scales

The Current and Childhood ADHD Symptoms Rating Scales (Barkley \& Murphy, 1998) are brief, selfreport screening questionnaires for assessment of adult ADHD. Questionnaire items reflect diagnostic criteria for ADHD as per the DSM-IV. These scales have high reliability and scale validity as indicated by significant correlations between self-report and spouse/parent ratings (Barkley \& Murphy, 1998).

\section{Conners' Adult ADHD Rating Scale, Screening Version}

The CAARS-S-SV (Conners et al., 1999) is a self-report measure of adult ADHD based on current scientific understanding of ADHD symptoms in adulthood. The CAARS-S-SV measures four dimensions: Inattention/Executive Function, Hyperactivity/Restlessness, Impulsivity/Emotional Lability, and Problems with Self-Concept (Conners et al., 1999).

Alien fruit creation task

Conceptual expansion was assessed via a creative generation task adapted from previous studies (Ward et al., 1999, 2002). Task instructions were similar to the creative condition of Ward et al. (2002); participants were told: "In this task, you will be asked to imagine, draw, and describe fruit that might exist on another planet that is very different from Earth. Be as creative and unusual as you can, and try not to duplicate fruit that exists on Earth. You will have 20 minutes to complete this activity." Conceptual expansion was measured using a procedure adapted from previous studies (Abraham et al., 2006; Kray et al., 2006). Two undergraduate research assistants, blind to participant condition and not involved in data collection, referred to the visual depiction and verbal description provided for each creation to determine the presence of parts (seeds/pits, stems) and properties (sweet, juicy) typical of Earth fruit (Tversky \& Hemenway, 1984; Ward et al., 1999), and atypical features, defined as parts or properties that are absent or extremely rare in

The ACT college readiness assessment is a standardized achievement test used for college admissions in the United States. Scores range from 1 to 36 , with a national average score of 21 .

2 Due to strict inclusion criteria, the final sample size $(n=26)$ fell short of the 28 participants per group recommended by a power analysis via G*Power software (Faul, Erdfelder, Lang \& Buchner, 2007), but the relative purity of the groups likely increased the sensitivity of ADHD/non-ADHD group comparisons. 
any variety of known Earth fruit. Atypical features were clustered into the following categories: (a) unusual appendages (e.g., hand, straw), (b) sensory organs (e.g., tongue, eyeball), (c) actions (e.g., lava shooting; combustible), and (d) effects on consumer (e.g., X-ray vision). For scoring purposes, typicality was the sum of typical features (0-4) and atypicality was the sum of atypical clusters (0-4). To evaluate originality, two research assistants who were blind to condition and hypothesis judged each creation's resemblance to Earth fruit on a 5-point scale (lower scores indicated greater similarity to Earth fruit). Agreement between raters was high $(\alpha=.899, p<.0001)$. Rater disagreements were resolved by obtaining a third rating and selecting the median.

Product label invention task

The constraining effect of recently activated knowledge (task examples) was examined via a product label invention task adapted from Kray et al. (2006). Participants were instructed as follows: "Imagine that you are a consultant for an advertising agency, and you've been asked to create names for three new products. Please create a name for each new product. You will see a list of examples for each category, but please do NOT use or copy any aspects of the examples provided. You will have 10 minutes to complete this task." The same instructions also appeared at the top of the task sheet, which listed the three categories (pain relievers, nuclear elements, and pasta) and for each category, six examples. Within each of the three categories, the labels shared two common endings (a letter or group of letters). For pain reliever, the examples were: Tylenol, Anacin, Aspirin, Bufferin, Panadol, and Midol (common endings -ol, -in). Nuclear element examples were radon, plutonium, argon, carbon, radium, and uranium (-on, -ium). Pasta label examples were spaghetti, lasagna, fettuccini, rotini, pastina, and rigatoni (-i, -a). Conformity to examples was defined as the presence of an example ending in the label invention for the corresponding category (e.g., an invented pasta label ending in the letter "a"). For each participant, the conformity score was the total number of label inventions that included an example ending. The appropriateness of label inventions was also evaluated by two undergraduate research assistants (blind to condition), using a procedure adapted from Kray et al. (2006). Each response was rated on a 5-point scale, according to how well it revealed the product type (pasta, pain reliever, and nuclear element). A higher value indicated that the label was more descriptive of product category. For instance, the responses "Pastanoodlini" and "Zigganoodles" were rated as very descriptive of the category pasta. Intra-class correlation coefficient indicated that raters were reliable $(\alpha=.886, p<.0001)$. In the event of rater disagreement, a third rater was consulted and the median value was taken. For each participant, the descriptiveness score was the median rating across the three product categories.

\section{EXPERIMENTAL PROCEDURE}

After providing informed consent, participants completed the alien fruit creation task and the product label task. Tasks were administered by research assistants who were blind to the condition of participants in an order counter-balanced across subjects. After the creative generation tasks, participants completed the CAARS-S-SV (ADHD rating scales).

\section{RESULTS}

For each creative generation task, performance of the ADHD and non-ADHD groups was compared via independent-samples $t$-tests. To control the family wise error rate, Holm's sequential Bonferroni method was applied to interpret statistical significance (Holm, 1979). The overall alpha level was set at .05 for each set of comparisons. For the alien fruit creation task, the variables of originality, typical features, and atypical features were compared as a function of ADHD group. This yielded reliable differences for originality, with the creations of the ADHD group rated as more original $(M=3.387, S D=0.983)$ compared to the nonADHD group $(M=2.77, S D=0.908), t(50)=-2.35, p=.023(d=0.645)$. The ADHD group also included more atypical features $(M=1.65, S D=0.977)$, relative to the non-ADHD group $(M=0.654$, $S D=0.629), t(50)=-4.39, p<.001(d=1.22)$. Typical features did not reliably differ between the ADHD group $(M=2.46, S D=1.27)$ and the non-ADHD group $(M=2.81, S D=1.17), t(50)=1.02, p=.311$ $(d=0.287)$. A second set of analyses for the product label invention task revealed that the labels created by the ADHD group included fewer task examples endings $(M=1.31, S D=1.12)$, compared to the nonADHD group $(M=2.08, S D=0.891), t(50)=2.74, p=.009(d=0.760)$. The category descriptiveness of the labels did not reliably differ between the ADHD group $(M=2.96, S D=0.824)$ and the non-ADHD group $(M=3.08, S D=0.845), t(50)=0.499, p=.620(d=0.144)$ (Figure 1$)$. 


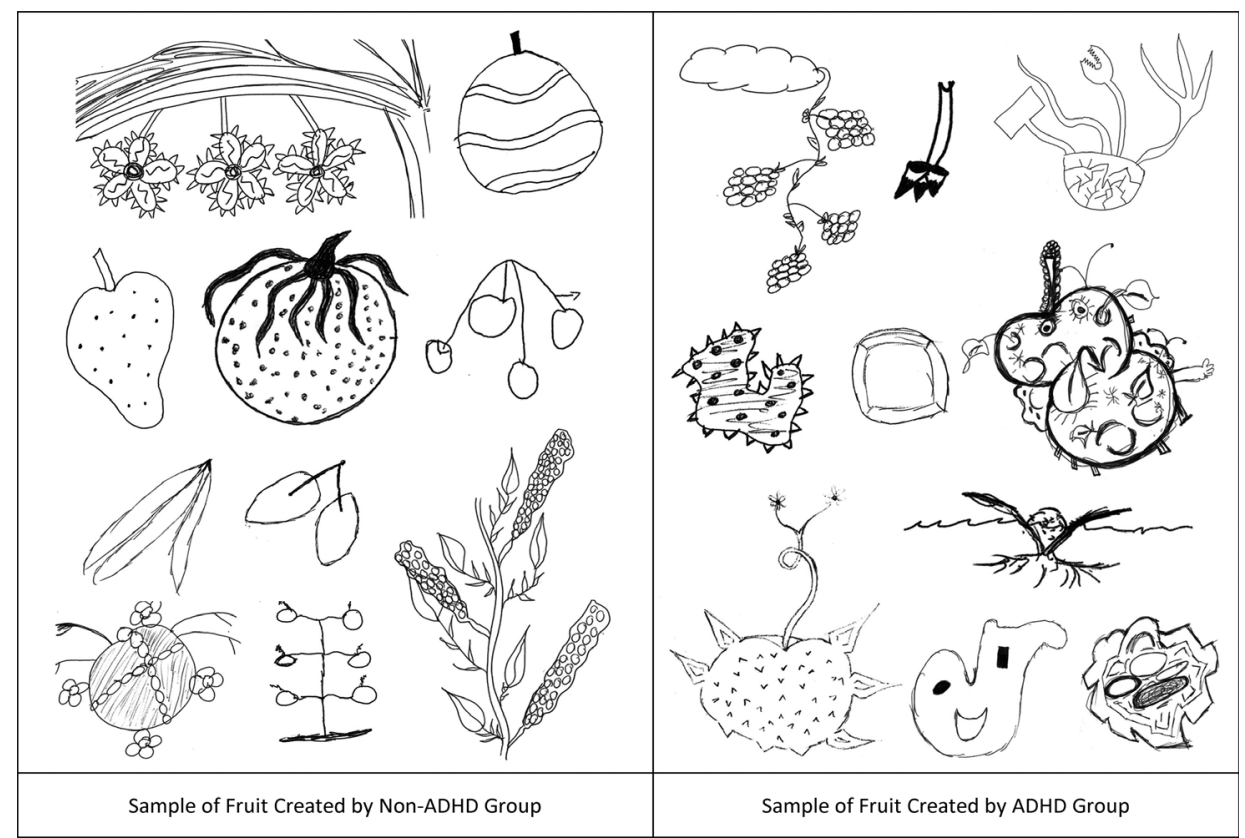

FIGURE 1. Sample of alien fruits created by non-ADHD and ADHD groups. ADHD, Attention Deficit Hyperactivity Disorder.

\section{DISCUSSION}

The present study tested the hypothesis that adults with ADHD would be less constrained by prior and recently activated knowledge during creative generation, relative to non-ADHD peers. The potential effect of prior knowledge was evaluated via the alien fruit task, wherein participants were asked to draw and describe fruit that might exist on a planet very different from Earth (Ward et al., 2002). Participants were explicitly instructed to be as creative and unusual as possible. This was intended to encourage conceptual expansion (thinking beyond traditional definition of "fruit"). To the extent that participants were constrained by prior knowledge of fruit schema, their creations were expected to include fewer atypical features and more closely resemble Earth fruit, as indicated by lower originality. As predicted, the ADHD group created alien fruit that was rated as more original and included more atypical features, relative to the non-ADHD group. To evaluate the effect of recently activated knowledge, participants were asked to invent labels for new products in specific categories. Examples were provided for each category, and participants were instructed to avoid copying any aspect of the task examples. The labels created by ADHD participants included fewer endings of task examples, compared to labels invented by non-ADHD participants; yet, the descriptiveness of labels did not differ between groups. The lack of conformity to task examples in the ADHD group was consistent with predictions and, together with results from the alien fruit task, supported the hypothesis that individuals with ADHD are less likely to be constrained by knowledge during creative generation.

The present findings are broadly consistent with previous research; adults with ADHD were more original on the alien fruit task, consistent with findings of higher originality on divergent thinking measures for ADHD individuals (White \& Shah, 2006, 2016). The present study also found support for higher conceptual expansion among adults with ADHD, in contrast to Abraham et al.'s (2006) finding for adolescents with ADHD. Yet, unlike Abraham et al. (2006), the present study did not use a composite index of conceptual expansion; previous studies suggested that atypical features are more indicative of creative performance than typical features, given that the latter are often associated with ceiling effects (Kray et al., 2006; Ward et al., 1999). Interestingly, the ADHD participants in the present study mirrored the performance of gifted adolescents in a study by Ward et al. (1999); like the ADHD group in the present study, gifted adolescents in 
Ward et al. (1999) designed alien fruits that were higher in originality and atypical features, yet similar in typical features, relative to a comparison group. Ward et al. (1999) suggested that typical features (e.g., seeds) may have been viewed as required to meet task objectives. Presumably, participants in the present study included typical features for the same reason; to indicate that the creation was indeed a fruit. This finding is notable, because it suggests that individuals with ADHD were able to be creative while staying within task parameters.

The creative generation tasks in the present study were intended to tap dissociable elements of creative cognition: conceptual expansion (alien fruit task) and the ability to overcome the constraining effects of recently activated knowledge (product label task) during creative generation (Abraham, 2014). These elements, along with divergent thinking, are critical components of creative cognition (Abraham \& Windmann, 2007). The present findings are therefore an important addition to the literature describing creative cognition in adults with ADHD. More generally, there may be an advantage of chaotic, unstructured cognition in creative generation; studies have demonstrated an increased ability to overcoming the constraining influence of task examples in children with ADHD (Abraham et al., 2006), individuals high in trait schizotypy (Abraham et al., 2007), and patients with schizophrenia (Abraham \& Windmann, 2008). Moreover, this commonality may have shared neurological underpinning; interference from the default mode network (SonugaBarke \& Castellanos, 2007). Nonetheless, overinclusive thinking that overly overinclusive may not yield viable, practical ideas (Eysenck, 1993).

The goal of "creative realism" requires a balance between the structured and chaotic elements of creative thinking (Finke, 1996). In the present study, ADHD participants created alien fruits that met implicit and definitional criteria for the category fruit, yet demonstrated imaginative divergence and originality. On the product label invention task, ADHD participants created labels that conformed less to task examples, yet were equally descriptive of product type, compared to labels invented by non-ADHD participants. Moreover, past research suggests that adults with ADHD are able to invent practical features in a realistic task of creative generation (White \& Shah, 2016). Thus, the relatively mild chaos associated with ADHD may be divergent, but not overly so.

Of course, an idea for a product is not the same as an actual creative product (Ward, 2004). According to the creative cognitive model, ideas represent "preinventive forms" which require transformational processes in order to manifest as an actual finished product (Ward, 2004; Ward et al., 1995). The later stages of the creative process are likely to require patience and delayed gratification; yet, individuals with ADHD tend to be motivated by immediate reward (Sonuga-Barke et al., 2008). Hence, people with ADHD may be less interested in the development or implementation of their creative ideas. In fact, research suggests that individuals with ADHD prefer the ideation (brainstorming) phase of the creative process over clarification of the problem space or development of ideas (White \& Shah, 2011). Nonetheless, idea generation is inherently valuable; perhaps the chaotic mind of an ADHD person paired with the structured mind of an ordered thinker would create synergy in the context of a joint project. Studies show that exposure to creative examples and solutions may heighten originality in the context of creative generation and problem solving (Agogué et al., 2014; Marsh et al., 1996; Perttula \& Sipilä, 2007). Might a person with ADHD influence the creativity of others, perhaps by widening conceptual space or disrupting the default structured approach to creative generation? Perhaps adults with ADHD could be creative catalysts in the workplace. While these are empirical questions, they are exciting possibilities that might be explored in future research.

\section{LIMITATIONS}

The present research has exciting potential theoretical and practical implications, but there are limitations that affect the interpretation and application of these findings. Primarily, the small sample size limited the power of this study and may have increased the risk of error. Thus, results should be viewed as preliminary. The relative purity of the present sample is both a strength and limitation of the present design; the lack of comorbidity and relatively high functioning participants in the ADHD group may have allowed for ADHD-related creative strengths to emerge, yet these findings may not generalize to all adults with ADHD. Adults with comorbid psychiatric conditions, learning difficulties, or more severe executive impairment were not represented in the current study. Moreover, the college students in the present study were relatively high functioning and may have possessed above-average intellect; this is noteworthy, because the link between disinhibition and originality may be positively affected by intelligence (Benedek, Franz, Heene \& Neubauer, 2012). Importantly, the present findings may not apply to individuals who are more clinically impaired (Paek, Abdulla \& Cramond, 2016). Finally, the creative generation tasks included in the present study were 
relatively simple and open-ended; perhaps complex problems or constrained tasks would increase reliance on task examples (Perttula \& Sipilä, 2007). Replication with a wider variety of measures and participants is recommended.

\section{CONCLUSION}

The results of this study provide further evidence that ADHD may be advantageous for certain types of creative thinking; specifically, divergent, unconstrained creative cognition. Future research might explore the potential contribution of the chaotic ADHD mind in the workplace. Research suggests that individual creativity is more likely to manifest as organizational creativity when leadership supports a culture of innovation (Stempfle, 2011), and many of the most innovation companies actively encourage "outside the box" thinking (Basadur, Runco \& Vega, 2000). By leveraging ADHD-related strengths and providing the necessary structure and support (Gupta \& Singhal, 1993), individuals and organizations alike may be able to unlock the imaginative and innovative potential of the ADHD mind.

\section{REFERENCES}

Abraham, A. (2014). Creative thinking as orchestrated by semantic processing vs. cognitive control brain networks. Frontiers in Human Neuroscience, 8, 95. https://doi.org/10.3389/fnhum.2014.00095.

Abraham, A., \& Windmann, S. (2007). Creative cognition: The diverse operations and the prospect of applying a cognitive neuroscience perspective. Methods, 42, 38-48. https://doi.org/10.1016/j.ymeth.2006.12.007.

Abraham, A., \& Windmann, S. (2008). Selective information processing advantages in creative cognition as a function of schizotypy. Creativity Research Journal, 20, 1-6. https://doi.org/10.1080/10400410701839819.

Abraham, A., Windmann, S., Daum, I., \& Güntürkün, O. (2005). Conceptual expansion and creative imagery as a function of psychoticism. Consciousness and Cognition, 14, 520-534. https://doi.org/10.1016/j.concog.2004.12.003.

Abraham, A., Windmann, S., McKenna, P., \& Güntürkün, O. (2007). Creative thinking in schizophrenia: The role of executive dysfunction and symptom severity. Cognitive Neuropsychiatry, 12, 235-258. https://doi.org/10.1080/13546800601046714.

Abraham, A., Windmann, S., Siefen, R., Daum, I., \& Güntürkün, O. (2006). Creative thinking in adolescents with attention deficit hyperactivity disorder (ADHD). Child Neuropsychology, 12, 111-123. https://doi.org/10.1080/09297040500320691.

Acar, S., \& Runco, M.A. (2012). Psychoticism and creativity: A meta-analytic review. Psychology of Aesthetics, Creativity, and the Arts, 6, 341-350. https://doi.org/10.1037/a0027497.

Agogué, M., Kazakçi, A., Hatchuel, A., Masson, P., Weil, B., Poirel, N., \& Cassotti, M. (2014). The impact of type of examples on originality: Explaining fixation and stimulation effects. Journal of Creative Behavior, 48, 1-12. https://doi.org/10.1002/jocb.37.

American Psychiatric Association (2013). Diagnostic and statistical manual of mental disorders (5th edn). Washington, DC: American Psychiatric Pub.

Barkley, R.A. (1997). Behavioral inhibition, sustained attention, and executive functions: Constructing a unifying theory of ADHD. Psychological Bulletin, 121, 65-94. https://doi.org/10.1037/0033-2909.121.1.65.

Barkley, R.A., \& Murphy, K.R. (1998). Attention-deficit hyperactivity disorder: A clinical workbook (pp. 90-94). New York: The Guilford Press.

Basadur, M., Runco, M.A., \& Vega, L.A. (2000). Understanding how creative thinking skills, attitudes and behaviors work together: A causal process model. Journal of Creative Behavior, 34, 77-100. https://doi.org/10.1002/j.2162-6057.2000.tb01203.x.

Beaty, R.E., Benedek, M., Silvia, P.J., \& Schacter, D.L. (2016). Creative cognition and brain network dynamics. Trends in Cognitive Sciences, 20, 87-95. https://doi.org/10.1016/j.tics.2015.10.004.

Benedek, M., Franz, F., Heene, M., \& Neubauer, A.C. (2012). Differential effects of cognitive inhibition and intelligence on creativity. Personality and Individual Differences, 53, 480-485. https://doi.org/10.1016/j.paid.2012.04.014.

Casakin, H.P., \& Goldschmidt, G. (2000). Reasoning by visual analogy in design problem-solving: The role of guidance. Environment and Planning B: Planning and Design, 27, 105-119. https://doi.org/10.1068/b2565.

Castellanos, F.X., \& Aoki, Y. (2016). Intrinsic functional connectivity in attention-deficit/hyperactivity disorder: A science in development. Biological Psychiatry: Cognitive Neuroscience and Neuroimaging, 1, 253-261. https://doi.org/10.1016/j.bpsc.2016.03.004.

Castellanos, F.X., Margulies, D.S., Kelly, C., Uddin, L.Q., Ghaffari, M., Kirsch, A., ... Sonuga-Barke, E.J. (2008). Cingulate-precuneus interactions: A new locus of dysfunction in adult attention-deficit/hyperactivity disorder. Biological Psychiatry, 63, 332337. https://doi.org/10.1016/j.biopsych.2007.06.025.

Castellanos, F.X., \& Proal, E. (2012). Large-scale brain systems in ADHD: Beyond the prefrontal-striatal model. Trends in Cognitive Sciences, 16, 17-26. https://doi.org/10.1016/j.tics.2011.11.007.

Chrysikou, E.G., \& Weisberg, R.W. (2005). Following the wrong footsteps: Fixation effects of pictorial examples in a design problem-solving task. Journal of Experimental Psychology: Learning, Memory, and Cognition, 31, 1134-1148. https://doi.org/10.1037/ 0278-7393.31.5.1134.

Conners, C.K., Erhardt, D., \& Sparrow, E. (1999). Conner's adult ADHD rating scales: Technical manual. New York: Multi-Health Systems Incorporated (MHS).

Eysenck, H.J. (1993). Creativity and personality: Suggestions for a theory. Psychological Inquiry, 4, 147-178. https://doi.org/10.1207/ s15327965pli0403_1. 
Eysenck, H.J. (1994). Creativity and personality: Word association, origence, and psychoticism. Creativity Research Journal, 7, 209216. https://doi.org/10.1080/10400419409534525.

Fassbender, C., Zhang, H., Buzy, W.M., Cortes, C.R., Mizuiri, D., Beckett, L., \& Schweitzer, J.B. (2009). A lack of default network suppression is linked to increased distractibility in ADHD. Brain Research, 1273, 114-128. https://doi.org/10.1016/j.brainres. 2009.02.070.

Faul, F., Erdfelder, E., Lang, A.G., \& Buchner, A. (2007). G* Power 3: A flexible statistical power analysis program for the social, behavioral, and biomedical sciences. Behavior Research Methods, 39, 175-191. https://doi.org/10.3758/BF03193146.

Finke, R.A. (1996). Imagery, creativity, and emergent structure. Consciousness and Cognition, 5, 381-393. https://doi.org/10.1006/cc og.1996.0024.

Finke, R.A., \& Bettle, J. (1996). Chaotic cognition: Principles and applications. Mahwah, NJ: Lawrence Erlbaum Associates.

Finke, R.A., Ward, T.B., \& Smith, S.M. (1992). Creative cognition: Theory, research, and applications. Cambridge, MA: MIT Press.

Goldschmidt, G. (2011). Avoiding design fixation: Transformation and abstraction in mapping from source to target. Journal of Creative Behavior, 45, 92-100. https://doi.org/10.1002/j.2162-6057.2011.tb01088.x.

Gupta, A.K., \& Singhal, A. (1993). Managing human resources for innovation and creativity. Research-Technology Management, 36, 41-48. https://doi.org/10.1080/08956308.1993.11670902.

Holm, S. (1979). A simple sequentially rejective multiple test procedure. Scandinavian Journal of Statistics, 6, 65-70. https://doi.org/ $10.2307 / 2337008$.

Jansson, D.G., \& Smith, S.M. (1991). Design fixation. Design Studies, 12, 3-11. https://doi.org/10.1016/0142-694X(91)90003-F.

Kray, L.J., Galinsky, A.D., \& Wong, E.M. (2006). Thinking within the box: The relational processing style elicited by counterfactual mind-sets. Journal of Personality and Social Psychology, 91, 33-48. https://doi.org/10.1037/0022-3514.91.1.33

Landau, J., \& Leynes, P.A. (2004). Manipulations that disrupt generative processes decrease conformity to examples: Evidence from two paradigms. Memory, 12, 90-103. https://doi.org/10.1080/09658210244000388.

Marsh, R.L., Landau, J.D., \& Hicks, J.L. (1996). How examples may (and may not) constrain creativity. Memory and Cognition, 24, 669-680. https://doi.org/10.3758/BF03201091.

Marsh, R.L., Ward, T.B., \& Landau, J.D. (1999). The inadvertent use of prior knowledge in a generative cognitive task. Memory and Cognition, 27, 94-105. https://doi.org/10.3758/BF03201216.

Nigg, J.T. (2001). Is ADHD a disinhibitory disorder? Psychological Bulletin, 127, 571-598. https://doi.org/10.1037//0033-2909.127.5. 571.

Paek, S.H., Abdulla, A.M., \& Cramond, B. (2016). A meta-analysis of the relationship between three common psychopathologiesADHD, anxiety, and depression-and indicators of little-c creativity. Gifted Child Quarterly, 60, 117-133. https://doi.org/10. 1177/0016986216630600.

Perttula, M., \& Sipilä, P. (2007). The idea exposure paradigm in design idea generation. Journal of Engineering Design, 18 , 93-102. https://doi.org/10.1080/09544820600679679.

Smith, S.M., Ward, T.B., \& Schumacher, J.S. (1993). Constraining effects of examples in a creative generation task. Memory and Cognition, 21, 837-845. https://doi.org/10.3758/BF03202751.

Sonuga-Barke, E.J., \& Castellanos, F.X. (2007). Spontaneous attentional fluctuations in impaired states and pathological conditions: A neurobiological hypothesis. Neuroscience and Biobehavioral Reviews, 31, 977-986. https://doi.org/10.1016/j.neubiorev.2007.02. 005 .

Sonuga-Barke, E.J., Sergeant, J.A., Nigg, J., \& Willcutt, E. (2008). Executive dysfunction and delay aversion in Attention Deficit Hyperactivity disorder: Nosologic and diagnostic implications. Child and Adolescent Psychiatric Clinics, 17, 367-384. https://d oi.org/10.1016/j.chc.2007.11.008.

Stempfle, J. (2011). Overcoming organizational fixation: Creating and sustaining an innovation culture. Journal of Creative Behavior, 45, 116-129. https://doi.org/10.1002/j.2162-6057.2011.tb01091.x.

Takeuchi, H., Taki, Y., Hashizume, H., Sassa, Y., Nagase, T., Nouchi, R., \& Kawashima, R. (2011). Failing to deactivate: The association between brain activity during a working memory task and creativity. NeuroImage, 55, 681-687. https://doi.org/10.1016/j. neuroimage.2010.11.052.

Thompson-Schill, S.L., Ramscar, M., \& Chrysikou, E.G. (2009). Cognition without control: When a little frontal lobe goes a long way. Current Directions in Psychological Science, 18, 259-263. https://doi.org/10.1111/j.1467-8721.2009.01648.x.

Tversky, B., \& Hemenway, K. (1984). Objects, parts, and categories. Journal of Experimental Psychology, 113, 169-193. https://doi. org/10.1037/0096-3445.113.2.169.

Ward, T.B. (1994). Structured imagination: The role of category structure in exemplar generation. Cognitive Psychology, $27,1-40$. https://doi.org/10.1006/cogp.1994.1010.

Ward, T.B. (2004). Cognition, creativity, and entrepreneurship. Journal of Business Venturing, 19, 173-188. https://doi.org/10.1016/ S0883-9026(03)00005-3.

Ward, T.B., Finke, R.A., \& Smith, S.M. (1995). Creativity and the mind: Discovering the genius within. https://doi.org/10.1007/978-14899-3330-0.

Ward, T.B., Patterson, M.J., \& Sifonis, C.M. (2004). The role of specificity and abstraction in creative idea generation. Creativity Research Journal, 16, 1-9. https://doi.org/10.1207/s15326934cri1601_1.

Ward, T.B., Patterson, M.J., Sifonis, C.M., Dodds, R.A., \& Saunders, K.N. (2002). The role of graded category structure in imaginative thought. Memory and Cognition, 30, 199-216. https://doi.org/10.3758/BF03195281. 
Ward, T.B., Saunders, K.N., \& Dodds, R.A. (1999). Creative cognition in gifted adolescents. Roeper Review, 21, 260-266. https://doi. org/10.1080/02783199909553973.

Ward, T.B., \& Wickes, K.N. (2009). Stable and dynamic properties of category structure guide imaginative thought. Creativity Research Journal, 21, 15-23. https://doi.org/10.1080/10400410802633376.

White, H.A. (2007). Inhibitory control of proactive interference in adults with ADHD. Journal of Attention Disorders, 11, 141-149. https://doi.org/10.1177/1087054706295604.

White, H.A., \& Shah, P. (2006). Uninhibited imaginations: Creativity in adults with attention-deficit/hyperactivity disorder. Personality and Individual Differences, 40, 1121-1131. https://doi.org/10.1016/j.paid.2005.11.007.

White, H.A., \& Shah, P. (2011). Creative style and achievement in adults with attention-deficit/hyperactivity disorder. Personality and Individual Differences, 50, 673-677. https://doi.org/10.1016/j.paid.2010.12.015.

White, H.A., \& Shah, P. (2016). Scope of semantic activation and innovative thinking in college students with ADHD. Creativity Research Journal, 28, 275-282. https://doi.org/10.1080/10400419.2016.1195655.

Youmans, R.J. (2011). Design fixation in the wild: Design environments and their influence on fixation. Journal of Creative Behavior, 45, 101-107. https://doi.org/10.1002/j.2162-6057.2011.tb01089.x.

Holly A. White, University of Michigan, University of Memphis

Correspondence concerning this article should be addressed to Holly White, Department of Psychology, University of Michigan Ann Arbor, 1004 East Hall, 530 Church St, Ann Arbor, MI 48109-1043.

E-mail: whiteha@umich.edu 